\title{
Ex vivo validation of a real-time multispectral endoscopic system for the detection and biopsy of bladder tumors
}

\author{
Britta Grüne ${ }^{1}$, Jan Rother ${ }^{2}$, Frank Waldbillig ${ }^{1}$, Ganapathy Chellappan ${ }^{2}$, Sabine Meessen ${ }^{3}$, \\ Bartłomiej Grychtol $^{2,4}$, Nikolaos C. Deliolanis, ${ }^{2,4}$, Christian Bolenz ${ }^{3}$, Maximilian C. Kriegmair ${ }^{1}$ \\ ${ }^{1}$ Department of Urology and Uro-Surgery, University Medical Center Mannheim, University of Heidelberg, Mannheim, Germany; ${ }^{2}$ Medical Faculty \\ Mannheim, University of Heidelberg, Mannheim, Germany; ${ }^{3}$ Department of Urology, University of Ulm, Ulm, Germany; ${ }^{4}$ Fraunhofer IPA - Project \\ Group for Automation in Medicine and Biotechnology (PAMB), Mannheim, Germany \\ Contributions: (I) Conception and design: B Grüne, J Rother, G Chellappan, MC Kriegmair; (II) Administrative support: All authors; (III) Provision of \\ study materials or patients: B Grüne, J Rother, G Chellappan, F Waldbillig; (IV) Collection and assembly of data: B Grüne, J Rother, G Chellappan; \\ (V) Data analysis and interpretation: All authors; (VI) Manuscript writing: All authors; (VII) Final approval of manuscript: All authors. \\ Correspondence to: Dr. med. Britta Grüne. Department of Urology and Uro-Surgery, University Medical Center Mannheim, University of Heidelberg, \\ Theodor-Kutzer-Ufer 1-3, D-68167 Mannheim, Germany. Email: britta.gruene@umm.de.
}

\begin{abstract}
Background: Real-time multispectral imaging (rMSI) simultaneously provides white light (WL), photodynamic diagnosis (PDD) images, and a real-time fusion of both. It may improve the detection of bladder tumors. However, rMSI has not been used for transurethral biopsy or resection so far. The aim of this ex vivo study was to test the feasibility of bladder tumor biopsies using the rMSI system and compare it to a conventional endoscopic system.
\end{abstract}

Methods: A 3D printed rigid bladder phantom was equipped with small and flat $(5 \mathrm{~mm} \times 1 \mathrm{~mm})$ mockbladder-tumors made of silicone and fluorescent Qdots655 (Thermo Fisher Scientific, Germany). Urologists $(n=15)$ were asked to perform a rigid cystoscopy and biopsy of all identified lesions $(n=6)$ using a prototype rMSI system and the Image1 S system (Karl Storz, Tuttlingen). Success rate and completion time were measured. The image quality of both systems and the usability of the rMSI system according to the system usability scale (SUS) were evaluated with a task-specific questionnaire.

Results: Tumor detection and biopsy rate were 100\% (90/90) for the rMSI system and 98.9\% (89/90) for the Image1 $\mathrm{S}$ system $(\mathrm{P}=0.3)$. The biopsy completion time did not differ significantly between the systems $(\mathrm{P}=0.48)$. Differentiation between healthy and suspect mucosa with the rMSI system was rated as comparable to the Image $1 \mathrm{~S}$ system by $53 \%$ of surgeons and as better by $33 \%$ of the surgeons. The median SUS score for the rMSI system was $87.5 \%$.

Conclusions: Accurate transurethral biopsies are feasible with the rMSI system. Furthermore, the rMSI system has an excellent SUS. This study paves the way to the first in-human transurethral resections of bladder tumors (TUR-B) using rMSI technology.

Keywords: Bladder cancer; cystoscopy; photodynamic diagnostic (PDD); multispectral; multiparametric cystoscopy (MP cystoscopy)

Submitted Nov 02, 2020. Accepted for publication Apr 04, 2021.

doi: $10.21037 /$ tau-20-1372

View this article at: http://dx.doi.org/10.21037/tau-20-1372

\section{Introduction}

Bladder cancer is the tenth most common cancer worldwide with 549,393 newly diagnosed cases in 2018 (1). Approximately $75 \%$ of bladder tumors are non-muscle- invasive at the time of diagnosis (2) and can be treated by repeated transurethral resection (TUR) and/or instillation therapies. Due to the high probability of recurrence (15$61 \% 1$ year and $31-78 \% 5$ years after TUR) (3), regular 
cystoscopic examinations are indispensable. The lifelong provision of follow-up care constitutes an enormous economic burden (4-6).

Although white light cystoscopy (WLC) remains the diagnostic standard for the detection of bladder tumors, flat cancerous lesions may be overlooked in up to $50 \%$ of patients (7). The most feared carcinoma in situ (CIS) is a high-grade, often multifocal non-muscle-invasive bladder cancer (NMIBC) (8), which has a high diseasespecific mortality in untreated patients (9). If not biopsied and further histopathologically examined, it can be misinterpreted, for example as an inflammatory bladder area (10) and remain undetected.

In recent years enhanced cystoscopic imaging techniques, like photodynamic diagnosis (PDD) and narrow-bandimaging (NBI) were developed to improve the diagnosis of bladder cancer. PDD has a significantly higher sensitivity than WLC for the detection of malignant tumors (92\% vs. $71 \%)(11)$, especially for high-grade tumors. The additional detection rate of PDD over WLC ranges between $17 \%$ and $78 \%$ in CIS patients (12). However, PDD has a relatively high rate of false positive results $(13,14)$ and its effect on reduction in recurrence and progression is still inconsistent (15-18).

To resolve the limitations of WLC and the enhanced cystoscopic systems, we recently established the real-time multispectral imaging (rMSI) technology in endourology. After the preclinical evaluation, including both in vivo (mouse model) and ex vivo trials (porcine bladder) (19), we used rMSI to perform a multiparametric (MP) cystoscopy for the detection of bladder tumors for the first time in human patients (20). By using an extended spectral resolution of reflected and fluorescent light, rMSI can obtain visual information that is not visible under white light (WL) (21). Different image modalities can be viewed at the same time, so that switching between them is not necessary. It is also possible to separate protoporphyrin IX (PpIX) fluorescence from autofluorescence and merge different images (WL, enhanced vascular contrast, PDD, PpIX fluorescence, autofluorescence) into a single MP image. Besides the assumption that MP imaging technology can increase the detection rate of bladder tumors, it is presumed that it can lead to a more complete TUR (20). Ahmadi and Daneshmand suggest that the greatest potential advantage of the rMSI system could be the minimization of the false positive rate and interobserver variability in image interpretation (22). So far, the rMSI system has only been used for cystoscopy and not during TUR of bladder tumors
(TUR-B). The feasibility and accuracy of biopsies and resection using the digitally processed images of the rMSI system remains unclear. Therefore, the aim of this ex vivo study was to test the handling of the novel rMSI system for biopsies of bladder tumors and compare it to a conventional endoscopic system.

We present the following article in accordance with the STARD reporting checklist (available at http://dx.doi. org/10.21037/tau-20-1372).

\section{Methods}

\section{Endoscopy systems}

\section{rMSI system}

The rMSI prototype system consists of a camera head, a control unit and a multi-LED light source (Omicron Laserage GmbH, Germany). A pendulum c-mount video adapter (asap endoscopic products $\mathrm{GmbH}$, Germany) is mounted to the camera to hold the endoscope. The system was mounted into an endoscopy tower. A PC was used to record the video output of the endoscopy system using an USB3 HDMI recorder (Magewell USB Capture HDMI Plus). Figure $1 A$ shows a photograph of the rMSI system. Although the arrangement of the different modalities offered by the rMSI can be changed from one up to four modalities on the screen at the same time, we kept the arrangement of the modalities as shown in Figure $1 B$ with the large MP image on the left side of the screen and the WL and the PDD modalities on the right side. The same configuration was used for all test urologists.

\section{Conventional PDD endoscopy platform}

An Image1 S system (Karl Storz GmbH \& Co., Tuttlingen, Germany) (Figure $2 A$ ), a conventional PDD-capable endoscopic system, served as a control in this ex vivo evaluation. Pressing on a foot pedal allowed to switch between WL and PDD images (Figure 2B).

\section{Endoscopes and light guides}

In all experiments a $30^{\circ} \mathrm{PDD}$-endoscope (Karl Storz GmbH \& Co., Tuttlingen, Germany) and liquid light guide (Karl Storz GmbH \& Co., Tuttlingen, Germany) were used. The endoscope was combined with a 26 French shaft. The biopsy forceps (27177 A, Karl Storz GmbH \& Co., Tuttlingen, Germany) was inserted into the bladder phantom through the working channel of the instrument. 


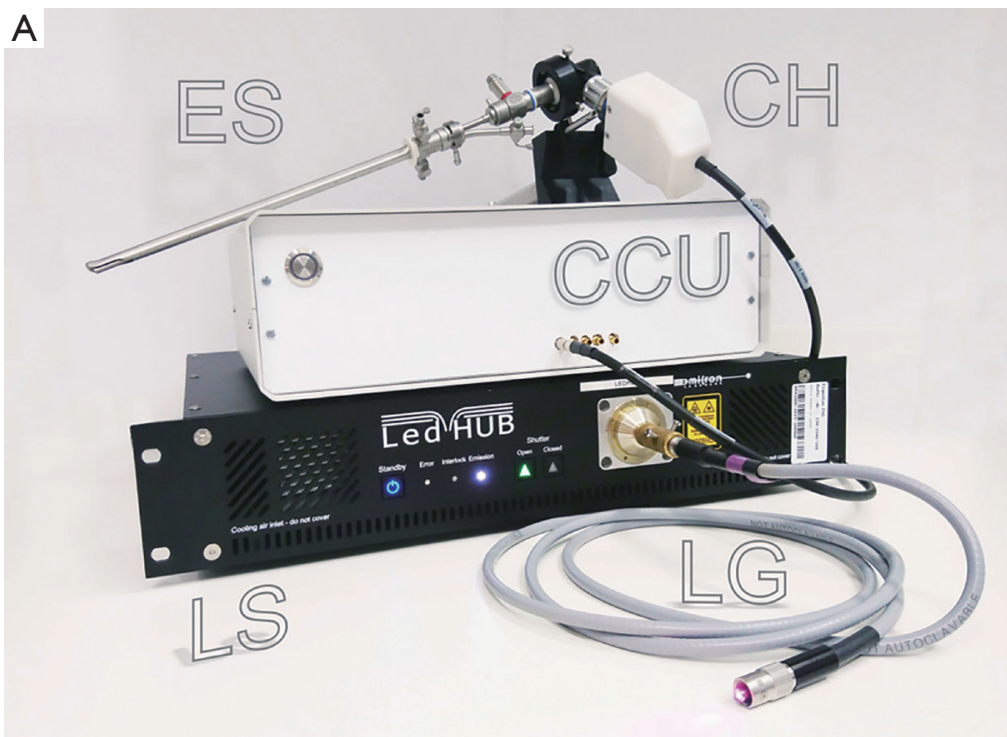

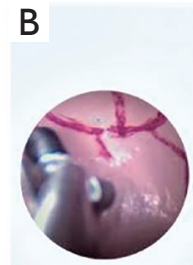

White light
rMSI cystoscopy

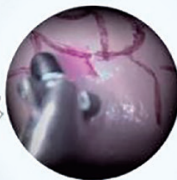

Real-time multiparametric

imaging

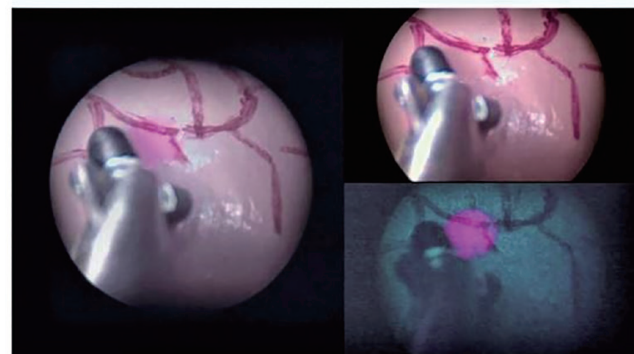

Figure 1 rMSI endoscopy system. (A) Image of the rMSI imaging system with the CCU controlling the CH and the LS. The image also shows the ES including shaft and the liquid LG. (B) Display of the imaging modalities of the rMSI system with the WL image, the PDD image and the MP image combining the aforementioned modalities. The image at the bottom shows screen captures. rMSI, real-time multispectral imaging; CCU, camera control unit; CH, camera head; LS, light source; ES, endoscope; LG, light guide; WL, white light; PDD, photodynamic diagnosis; MP, multiparametric.
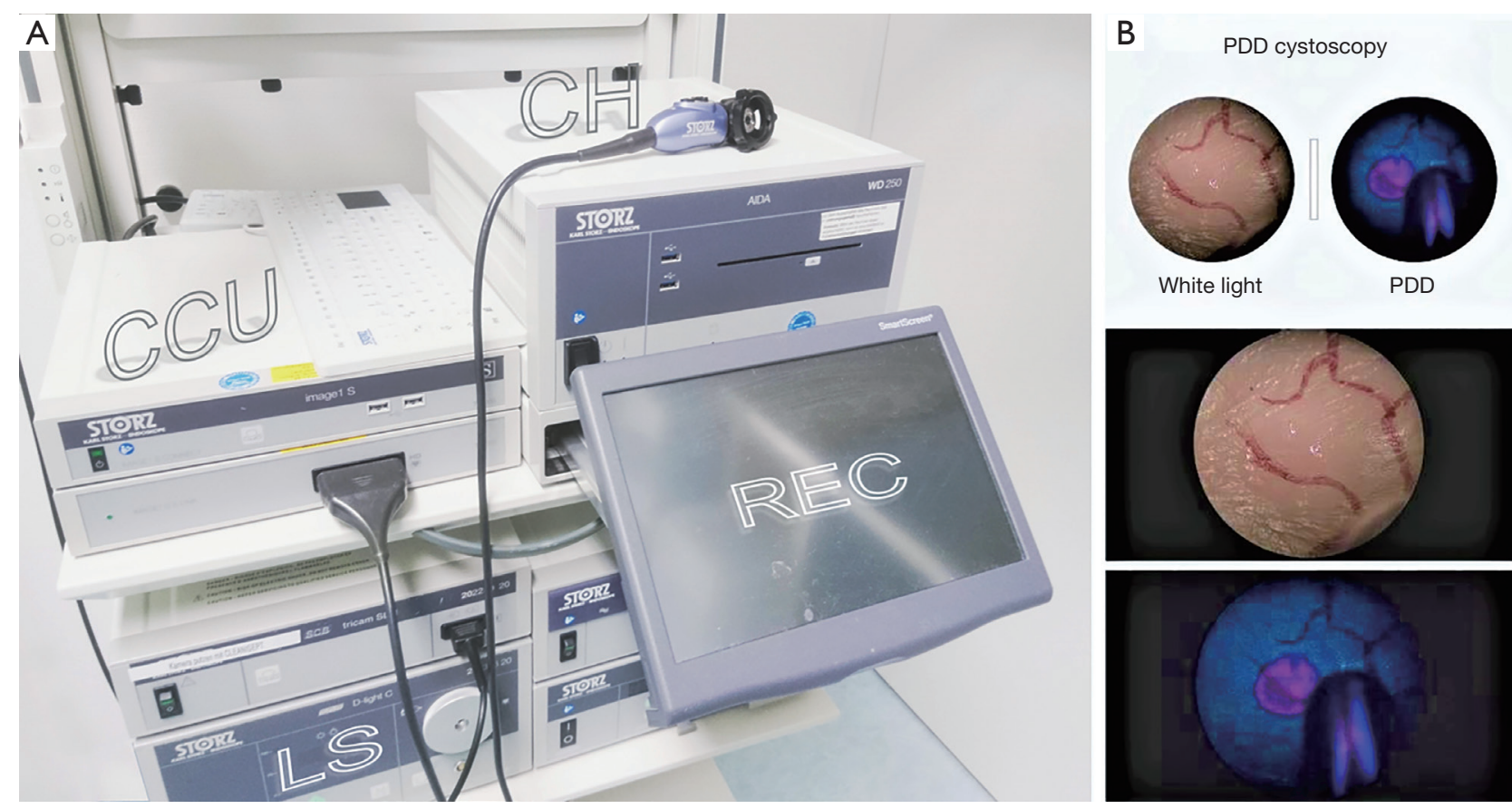

Figure 2 Commercial endoscopy system. (A) Image of the commercial endoscopy system with the CCU controlling the CH. The image also shows the commercial REC and the LS. (B) Display of the imaging modalities of the PDD system. The PDD system can be switched between the WL image and the PDD modality by means of a foot switch. The images at the bottom shows screen captures. CCU, camera control unit; CH, camera head; LS, light source; REC, recorder unit; PDD, photodynamic diagnosis; WL, white light. 


\section{Experimental setup}

\section{Bladder phantom}

A 3D printed polymeric bladder phantom based on data extracted and segmented from original high-resolution computed tomography data-sets was used for the experiment. The inside of the phantom was painted in a bladder mucosa-like color. Blood vessels were simulated by fine curled lines in red (Figure $3 A$ ). The phantom was mounted on a rigid stand (Figure $3 B$ ). The endoscope could be brought into the bladder phantom through a hole $(2 \mathrm{~cm}$ in diameter) at the bottom of its front side.

\section{Fluorescent bladder lesions}

To simulate fluorescent suspicious flat lesions, we generated small patches, $5 \mathrm{~mm}$ in diameter and $1 \mathrm{~mm}$ thick, using silicone mixed with PpIX-mimicking Qdots655 (Qdot ${ }^{\circledR} 655$ $\mathrm{ITK}^{\mathrm{TM}}$ organic quantum dots). We placed eight silicone patches on the inside of the bladder phantom. Three of the eight patches had a low concentration of Qdots655 $(5.8 \mathrm{nM})$ and three a high concentration $(20 \mathrm{nM})$ (ratio 1:3.45). Two silicone patches without Qdots655 were used as control. The patch locations, defined in advance, were unique to each endoscopic system (Figure 3C). Care was taken to ensure that the distribution of patches for the two systems were comparable in terms of ease of accessibility. In the first three trials, we observed that the patches on the ceiling of the model were difficult to remove with the forceps. Therefore, in subsequent studies all patches were placed in the lower half of the bladder model.

\section{Experimental procedure}

Overall, 15 urological surgeons with varying levels of experience independently took part in the prospective $e x$ vivo validation. Before the experiment, each surgeon was given the opportunity to familiarize her/himself with the respective cystoscope and the bladder phantom for five minutes. Furthermore, each surgeon performed one training cystoscopy including biopsy with the rMSI endoscopic system and one with the conventional endoscopic system. The following tasks were given to the surgeons: (I) perform whole bladder cystoscopy using PDD for detection of all fluorescent lesions of the bladder phantom, (II) extract all fluorescent lesions using biopsy forceps. The total number of lesions was unknown to the surgeons. The time until the surgeon had extracted all fluorescent lesions from the bladder and the time until the surgeon was sure that there were no more fluorescent lesions in the bladder were measured respectively. Detection and biopsy rate of the fluorescent lesions were also recorded.

When using the PDD system the surgeons were asked to enter the bladder in WL mode in accordance with the standard clinical procedure. Once inside the bladder, the surgeons were free to switch between PDD mode and WL. No switching between modes was required with the rMSI system.

\section{System usability}

After the experiment, each surgeon completed a customized task-specific questionnaire. The questionnaire evaluated the following four parameters: quality of the WL image, quality of the PDD image, subjective ability to differentiate between fluorescent and non-fluorescent signal and handling. For the conventional endoscopic system, a sixpoint Likert scale was used, with 1 denoting "very good" and 6 "very bad". For the rMSI system a four-point Likert scale was used to evaluate these parameters in comparison to the conventional system, with 1 denoting "better than the conventional system", 2 denoting "comparable", 3 "inferior, but sufficient" and 4 "worse and should be improved". By means of open-ended questions, the surgeons could indicate what they liked and disliked about the rMSI system.

Additionally, the questionnaire included the system usability scale (SUS) survey, which consists of 10 statements (alternately positive and negative) presented in a form of a five-point Likert scale, ranging from 1 (strongly disagree) to 5 (strongly agree). Based on this survey, the SUS score, a single score on a scale from 0 (worst conceivable application) to 100 (best conceivable application), can be calculated. The cut-off value for a system with above average usability is 68 . The SUS is a well-established and validated usability score $(23,24)$.

\section{Statistical analysis}

Statistical analysis was performed using SAS (JMP ${ }^{\circledR}$, version ${ }^{\circ} 14$. SAS Institute Inc., Cary, NC, USA). The Wilcoxon signed-rank test was used for group comparison (Image1 S vs. rMSI). Level of significance was defined as 0.05 . The first three attempts where lesions were placed also on the ceiling of the phantom were excluded from the analysis of the time taken to complete the tasks. The results of the questionnaires were evaluated qualitatively. 


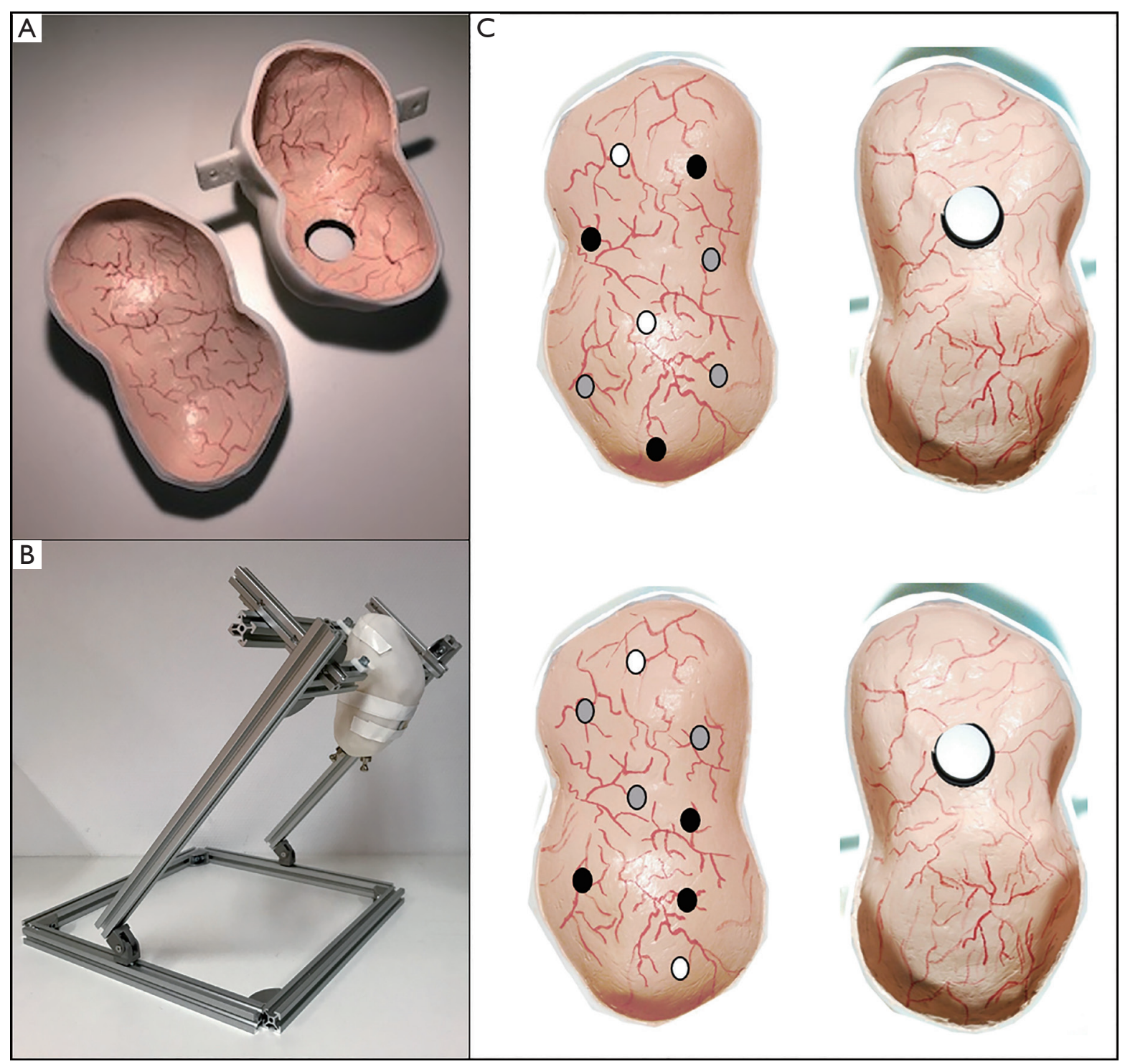

Figure 3 Experimental setup. (A) 3D-printed rigid bladder phantom. (B) Bladder phantom on a metallic stand. (C) Scheme of lesion positions (black: high concentrated lesion, grey: low concentrated lesion, white: control lesion) top: lesion positions for rMSI system, bottom: lesion positions for Image1 S system. rMSI, real-time multispectral imaging.

\section{Ethics statement}

Because neither human nor animal subjects were involved in this ex vivo study, approval by an ethics committee was not needed. The participation of urologists in the study was voluntary, so no written informed consent was obtained.

\section{Results}

The ex vivo trial was performed by 15 urologists with a median (interquartile range) age of 30 (27.3-31.8) years. Four participants (26.7\%) were female and 11 (73.3\%) were male. Two-thirds of them $(n=10)$ were residents and one-third $(\mathrm{n}=5)$ were board certified urologists. They had previously performed a median of 200 [20-500] WLC and 15 [0-60] PDD-cystoscopies.

\section{Biopsy performance}

Detection and biopsy rate of fluorescent lesions were 
Table 1 Performance of biopsy

\begin{tabular}{|c|c|c|c|}
\hline Outcome parameter & Image1 S & rMSI & $P$ value $[n]$ \\
\hline Low fluorescence & $44 / 45$ & $45 / 45$ & \\
\hline High fluorescence & $45 / 45$ & $45 / 45$ & \\
\hline Time till removal of the last lesion (sec.) & $176.2(78.7)^{\star}$ & $187.2(157.0)^{*}$ & $0.48[12]$ \\
\hline
\end{tabular}

*, values reported as mean (standard deviation). rMSI, real-time multispectral imaging.

98.9\% (89/90) for the Image1 S system and 100\% (90/90) for the rMSI System $(\mathrm{P}=0.3)$. No surgeon biopsied a nonfluorescent control lesion. The mean time (standard deviation) to complete biopsy of all lesions was $176.2 \mathrm{~s}$ (78.7) for the Image1 $S$ and $187.2 \mathrm{~s}$ (157.0) for the rMSI system $(\mathrm{P}=0.48)$. The corresponding values for time taken by surgeons to be certain of a complete biopsy were $260.1 \mathrm{~s}$ (104.7) and $259.5 \mathrm{~s}(151.3)(\mathrm{P}=0.85)$, respectively (Table 1). The execution times did not differ significantly between residents and urologists [time till removal of the last lesion for the Image1 S system: residents 195.6 (83.1), urologists 137.5 (31.7), $\mathrm{P}=0.24$; time till removal of the last lesion for the rMSI system: residents 205.9 (178.9), urologists 150 (40.6), $\mathrm{P}=0.58]$.

\section{System evaluation}

The evaluation of the rMSI endoscopic system resulted in a SUS score of $87.5 \%$ (Figure $4 A, B$ ). According to this value, the usability of the system is classified as excellent on the SUS scale ranging from 0 to 100 (Figure $4 B$ ). The box-plot chart (Figure 4C) shows the scores of the ten SUS statements on the five-point Likert scale.

Evaluation of the image quality of the WL and PDD images, the subjective ability to differentiate between fluorescence and non-fluorescence and the handling for Image $1 \mathrm{~S}$ and $\mathrm{rMSI}$ in comparison is shown in Figure 5. The surgeons rated all four parameters with the Image $1 \mathrm{~S}$ system as good or very good. Quality of the WL image of the rMSI system was rated by $80 \%$ of the physicians as comparable to the Image $1 \mathrm{~S}$ system and by $13 \%$ as better. Quality of the PDD image of the rMSI system was comparable to that of the conventional system for almost half of the respondents $(n=7 ; 47 \%)$ and better for one-third $(n=5$; $33 \%)$. For one-fifth $(\mathrm{n}=3 ; 20 \%)$ it was worse, but sufficient. The surgeons made a similar assessment regarding the ability to distinguish between fluorescent lesions and the "normal bladder mucosa". For 5 surgeons (33\%) this was better with the rMSI system and for 8 surgeons (53\%) it was comparable while 2 surgeons (13\%) felt it was worse, but sufficient. Sixty percent of the respondents felt that the handling of the rMSI system was comparable to that of the conventional system. Two respondents (13\%) found it better, $3(20 \%)$ worse but sufficient and 1 (7\%) worse and in need of improvement.

The answers to the questions "what do you like about the rMSI system?" and "what do you dislike about the rMSI system?" are shown in Figure 6.

\section{Discussion}

The promising results of our recent studies $(19,20)$, in which we applied rMSI technology for the first time in endourology, suggest that this novel approach may significantly improve the detection of bladder cancer. Using the images recorded with the rMSI system, two independent observers were able to identify all malignant lesions of ten patients on MP images. However, tumor resection was performed using a conventional endoscopic system. Accordingly, the application of the rMSI system during a tumor biopsy or tumor resection is still to be examined and compared to an existing and established endoscopic system. To the best of our knowledge this is the first study investigating the usability of a rMSI endoscopic system during a bladder tumor biopsy. Several preclinical studies (25-28) evaluated and proved the usability and feasibility of novel endoscopic systems ex vivo, thus paving the way for human trials.

For this ex vivo trial, we used a 3D printed polymeric bladder phantom. Since existing bladder models are scarce, expensive and different from genuine bladder anatomy, this phantom was developed with the aim of improving 
A

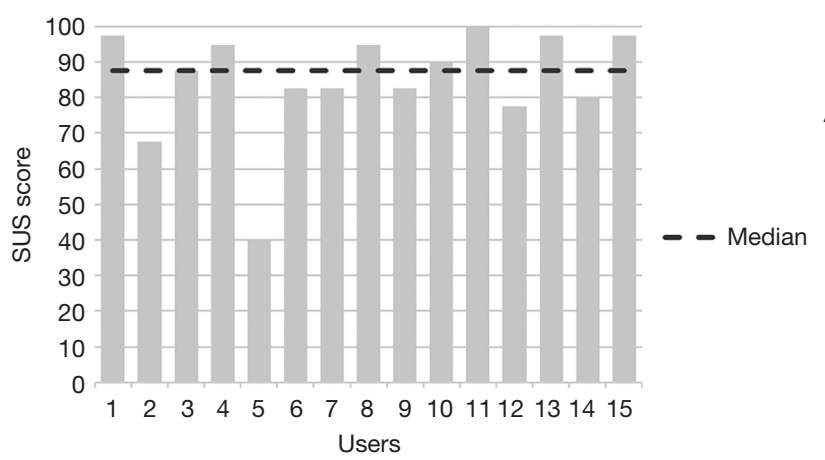

B

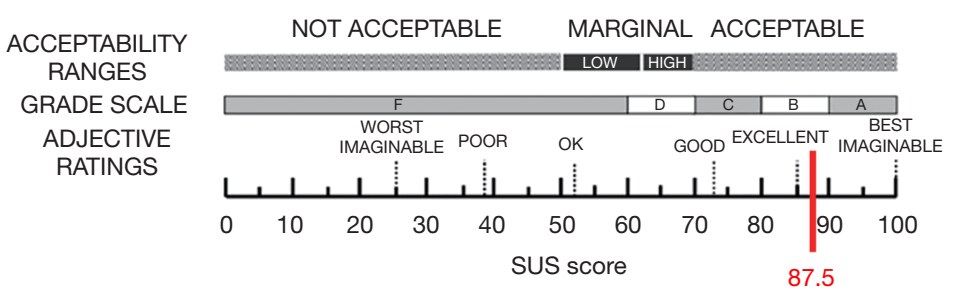

C

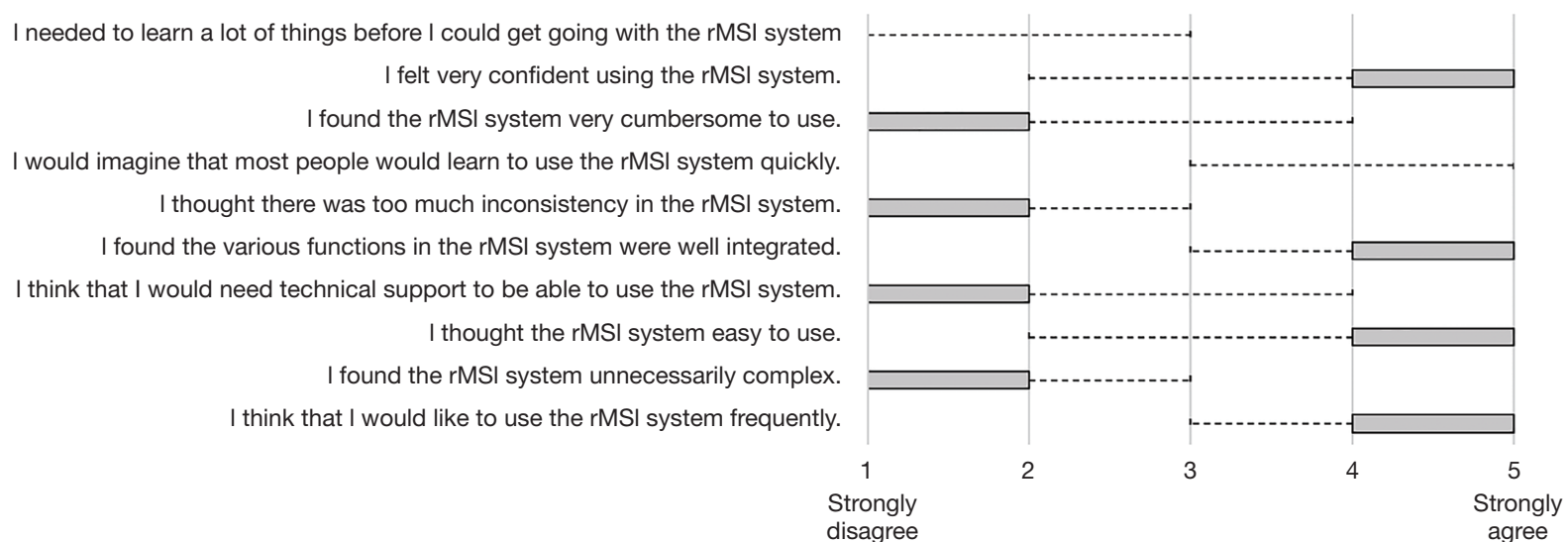

Figure 4 System usability. (A) SUS score for the rMSI system of each user. (B) Median SUS score for the rMSI system on the SUS scale. (C) Box-plot chart: scores of the ten SUS statements. SUS, system usability scale; rMSI, real-time multispectral imaging.

endourological training (29). This phantom, which has been used in two recently published studies $(28,29)$, has proven to be a good model with anatomically correct and realistic conditions. It is easy to produce, robust and functionally reproducible (29).

Operating surgeons assigned the rMSI system with a median SUS score of $87.5 \%$ (minimum rate required for good usability is $68 \%$ ) (24), which means that the system has an excellent usability in cystoscopy and biopsy of suspicious bladder lesions. With a detection rate of $100 \%$, the rMSI system also proved to be adequate for the detection of flat suspicious bladder lesions under the given experimental conditions. In fact, its detection and biopsy rate were slightly higher than those of the conventional system $(98.9 \%, \mathrm{P}=0.3)$. In their systematic review and metaanalysis Rink et al., 2013 report on a CIS detection rate of $46-100 \%$ using PDD cystoscopy (16). According to Mowatt et al., 2011, the PDD sensitivity in biopsy is $93 \%$ (11).
The comparison of these detection rates with those of the Image $1 \mathrm{~S}$ system (98.9\%) indicates that the ex vivo test conditions were somewhat easier for the surgeons than in reality. This could be a consequence of the clearer field of view for the surgeons due to the absence of intravesical bleeding or turbid urine as well as a greater mobility of the endoscope in the phantom due to the absence of subvesical obstructions.

Among the most liked features of the rMSI system mentioned in the open-ended questions were the simultaneous display of several image modalities (7/15 respondents) and the consequent absence of switching (3/15 respondents). Although some surgeons stated that the latter feature was very convenient and subjectively time saving, we did not find a difference of operation time between the two systems in terms of either the 'time till removal of last lesion' $(\mathrm{P}=0.48)$ or the 'time to surgeon's certainty of a complete biopsy' $(\mathrm{P}=0.85)$. A possible explanation for this 


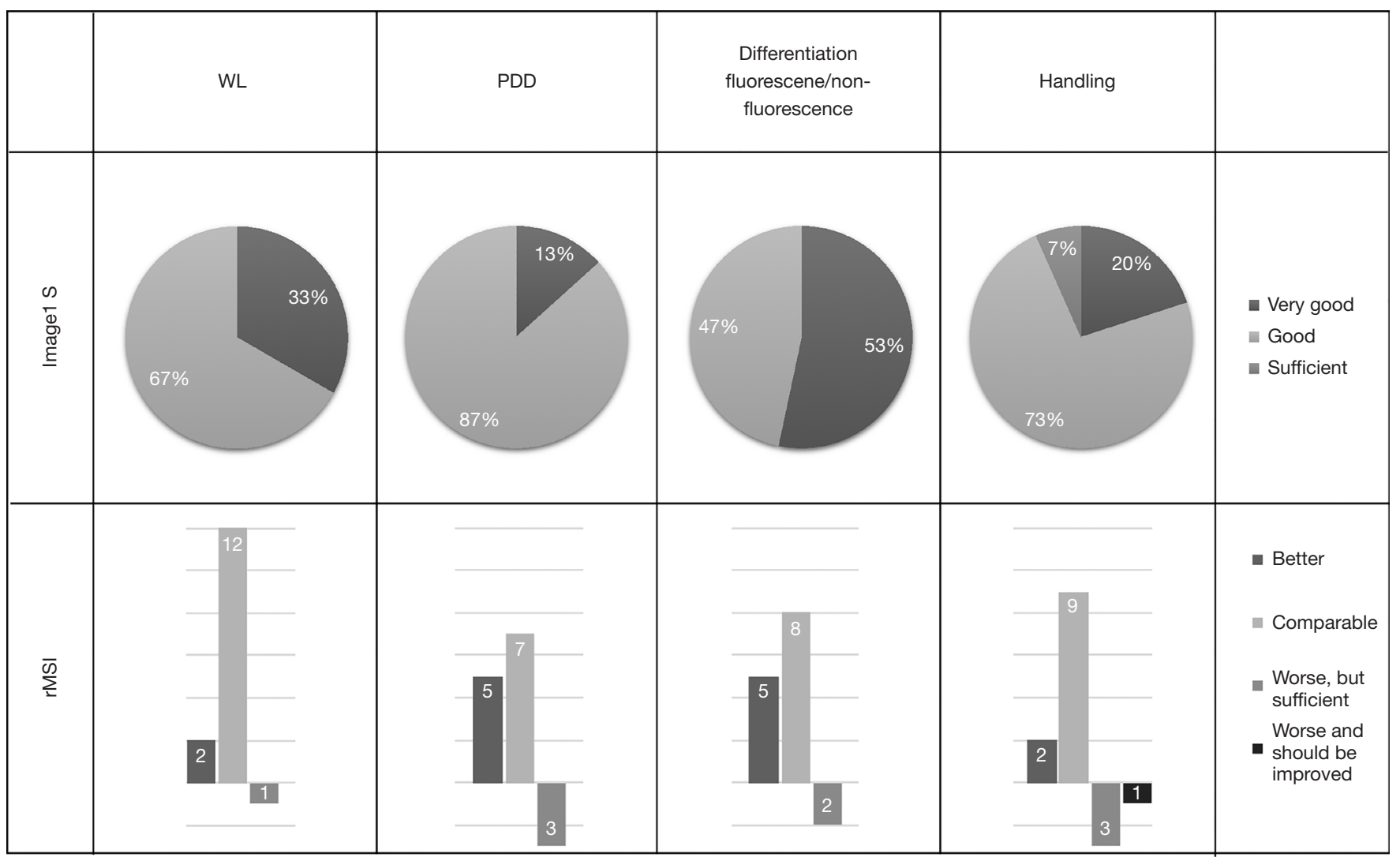

Figure 5 System evaluation-image quality and handling. Surgeons evaluation of the image quality of WL and PDD, feasibility to differentiate between fluorescence and non-fluorescence and handling for Image1 S and rMSI in comparison. WL, white light; PDD, photodynamic diagnosis; rMSI, real-time multispectral imaging.

What do you like about the rMSI system?

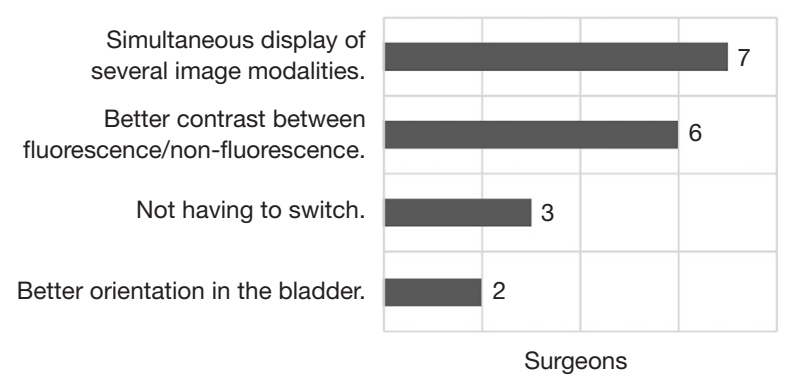

What do you dislike about the rMSI system?

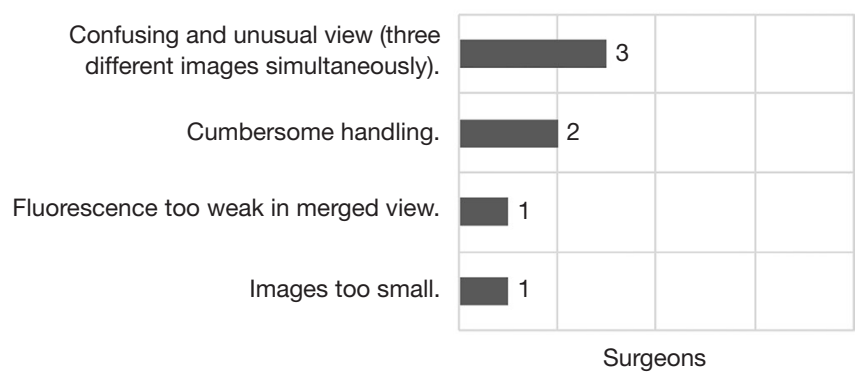

Figure 6 System evaluation-open-ended questions. Answers to the open-ended questions "what do you like about the rMSI system?" and "what do you dislike about the rMSI System?". rMSI, real-time multispectral imaging.

could be that the majority of surgeons were very experienced and familiar with PDD cystoscopy and therefore performed the entire biopsy with the Image1 S system in PDD mode without switching. Some surgeons indicated that PDD image was considerably brighter and the contrast between fluorescent lesions and bladder wall was much stronger under the experimental conditions than in reality und thus a switch to WL was not necessary for the biopsy. In a human urinary bladder, the view in PDD mode is usually too dark to perform a safe tumor biopsy or resection, so that 
after tumor detection via PDD, switching to $\mathrm{WL}$ is often necessary. Additionally, bleeding, which can occur after a biopsy or resection, can weaken the PDD signal so that the surgeon loses orientation within the bladder. Here, the simultaneous display of WL, PDD and merged image of the rMSI system could possibly offer a relevant advantage under in vivo conditions. When asked what they liked about the rMSI system, half of the surgeons also answered that they liked the simultaneous display of images. Even under ex vivo conditions, two surgeons stated that they felt they could better orient themselves in the bladder with the rMSI system. These considerations suggest that the rMSI system may allow faster tumor biopsy/resection under real in vivo conditions. Although a long procedure time is not the most important predictive factor for postoperative complications after TUR-B, it is nevertheless relevant (30).

The quality of the PDD image as well as the ability to distinguish between fluorescence and non-fluorescence was rated by $50 \%$ of the surgeons as comparable to the Image1 S system and by one-third as better. Six respondents specifically commented on the better contrast between fluorescence and non-fluorescence in the open-ended questions. This evaluation further supports the conclusion that the rMSI system may enable a higher detection rate of flat fluorescent bladder lesions compared to a conventional PDD endoscope.

The handling of the rMSI device was rated by $7 \%$ of the surgeons as worse and in need of improvement. Likewise, two respondents stated in open-ended answers that they did not like the awkward handling of the rMSI endoscope. It must be acknowledged that the ergonomics of the rMSI system are not refined and the camera head is significantly larger and heavier than that of the Image1 S system. However, a miniaturization of the camera head is technically possible and already planned to a greater extent. While half of the investigators stated that they liked the simultaneous display of several images, three of them named as their main criticism that this view was initially confusing and unfamiliar. However, it should be noted that these three examiners had no or very little experience with PDD cystoscopy.

The present study has several limitations, which are partially attributed to the ex vivo test conditions, as described in more detail below. In the first three trials the surgeons found it challenging to access the lesions placed on the top of the bladder phantom due to its rigid construction. This necessitated the change in the placement of the lesions for better accessibility and the consequent exclusion of the first three trials from the analysis of task duration. Since actual tumors may also be localized in bladder areas that are difficult to reach, the usability of the rMSI system should be evaluated for biopsies or resections of these challenging tumors in humans in the future. As discussed previously, vision in PDD mode in this study was significantly brighter compared to reality in a human bladder, most likely due to the stronger reflection of light from the rigid bladder wall. Therefore, an appropriate comparison of the endoscopy systems is only possible to a limited extent. Additionally, the bladder lesions fluoresced comparatively strongly and looked very homogeneous, so that a more precise assessment by the surgeon was not necessary. The fact that time was measured during the biopsy probably put the surgeons under time pressure and may have biased the results. Of course, a comparison of the rMSI system with an existing endoscopic system in vivo is essential. A further limitation of the study is the small number of investigators and cystoscopies performed. Due to the different devices and different views on the screen of the two endoscopic systems, the experiment could not be blinded. For this reason, a verification bias cannot be excluded. However, the systems were used in alternating order to avoid a possible influence of experience gain on the results.

\section{Conclusions}

The rMSI prototype allows comparable performance in biopsy of bladder lesions to a conventional PDD system and exhibits excellent usability. Minor concerns about handling are expected to be addressed with improvements in ergonomics. Simultaneous display of PDD and WL images is well accepted by the surgeons and may improve detection of bladder cancer and simplify biopsies in the future.

\section{Acknowledgments}

The authors are very grateful to all urologists, who participated in the study.

Funding: This work was supported by the funding from the German Ministry for Education and Research \{GO-Bio Project [031B0219]\}. The sponsor played no direct role in the study.

\section{Footnote}

Reporting Checklist: The authors have completed the STARD 
reporting checklist. Available at http://dx.doi.org/10.21037/ tau-20-1372

Data Sharing Statement: Available at http://dx.doi. org/10.21037/tau-20-1372

Peer Review File: Available at http://dx.doi.org/10.21037/ tau-20-1372

Conflicts of Interest: All authors have completed the ICMJE uniform disclosure form (available at http://dx.doi. org/10.21037/tau-20-1372). JR, GC, BG, CB and NCD report grants from the German Ministry for Education and Research (GO-Bio Project 031B0219). NCD is a coinventor in a patent WO2015185661A1 family. NCD and BG are shareholders of technology start-up "Thericon GmbH" with the aim to commercialize multispectral imaging technology. The other authors have no conflicts of interest to declare.

Ethical Statement: The authors are accountable for all aspects of the work in ensuring that questions related to the accuracy or integrity of any part of the work are appropriately investigated and resolved. Because neither human nor animal subjects were involved in this ex vivo study, approval by an ethics committee was not needed. The participation of urologists in the study was voluntary, so no written informed consent was obtained.

Open Access Statement: This is an Open Access article distributed in accordance with the Creative Commons Attribution-NonCommercial-NoDerivs 4.0 International License (CC BY-NC-ND 4.0), which permits the noncommercial replication and distribution of the article with the strict proviso that no changes or edits are made and the original work is properly cited (including links to both the formal publication through the relevant DOI and the license). See: https://creativecommons.org/licenses/by-nc-nd/4.0/.

\section{References}

1. Bray F, Ferlay J, Soerjomataram I, et al. Global cancer statistics 2018: GLOBOCAN estimates of incidence and mortality worldwide for 36 cancers in 185 countries. CA Cancer J Clin 2018;68:394-424. Erratum in: CA Cancer J Clin 2020;70:313.

2. Burger M, Catto JW, Dalbagni G, et al. Epidemiology and risk factors of urothelial bladder cancer. Eur Urol
2013;63:234-41.

3. Sylvester RJ, van der Meijden AP, Oosterlinck W, et al. Predicting recurrence and progression in individual patients with stage Ta T1 bladder cancer using EORTC risk tables: a combined analysis of 2596 patients from seven EORTC trials. Eur Urol 2006;49:466-5; discussion 475-7.

4. Mossanen M, Gore JL. The burden of bladder cancer care: direct and indirect costs. Curr Opin Urol 2014;24:487-91.

5. Leal J, Luengo-Fernandez R, Sullivan R, et al. Economic burden of bladder cancer across the European Union. Eur Urol 2016;69:438-47.

6. Wong MCS, Fung FDH, Leung C, et al. The global epidemiology of bladder cancer: a joinpoint regression analysis of its incidence and mortality trends and projection. Sci Rep 2018;8:1129.

7. Zaak D, Hungerhuber E, Schneede P, et al. Role of 5 -aminolevulinic acid in the detection of urothelial premalignant lesions. Cancer 2002;95:1234-8. Erratum in: Cancer 2002;95:2580.

8. Sylvester RJ, van der Meijden A, Witjes JA, et al. Highgrade Ta urothelial carcinoma and carcinoma in situ of the bladder. Urology 2005;66:90-107.

9. Shariat SF, Palapattu GS, Amiel GE, et al. Characteristics and outcomes of patients with carcinoma in situ only at radical cystectomy. Urology 2006;68:538-42.

10. Subiela JD, Rodríguez Faba O, Guerrero Ramos F, et al. Carcinoma in situ of the urinary bladder: a systematic review of current knowledge regarding detection, treatment, and outcomes. Eur Urol Focus 2020;6:674-82.

11. Mowatt G, N'Dow J, Vale L, et al. Photodynamic diagnosis of bladder cancer compared with white light cystoscopy: systematic review and meta-analysis. Int J Technol Assess Health Care 2011;27:3-10.

12. Kausch I, Sommerauer M, Montorsi F, et al. Photodynamic diagnosis in non-muscle-invasive bladder cancer: a systematic review and cumulative analysis of prospective studies. Eur Urol 2010;57:595-606.

13. Draga RO, Grimbergen MC, Kok ET, et al. Photodynamic diagnosis (5-aminolevulinic acid) of transitional cell carcinoma after bacillus Calmette-Guérin immunotherapy and mitomycin $\mathrm{C}$ intravesical therapy. Eur Urol 2010;57:655-60.

14. Ray ER, Chatterton K, Khan MS, et al. Hexylaminolaevulinate fluorescence cystoscopy in patients previously treated with intravesical bacille CalmetteGuérin. BJU Int 2010;105:789-94.

15. Grossman HB, Stenzl A, Fradet Y, et al. Long- 
term decrease in bladder cancer recurrence with hexaminolevulinate enabled fluorescence cystoscopy. J Urol 2012;188:58-62.

16. Rink M, Babjuk M, Catto JW, et al. Hexyl aminolevulinateguided fluorescence cystoscopy in the diagnosis and follow-up of patients with non-muscle-invasive bladder cancer: a critical review of the current literature. Eur Urol 2013;64:624-38.

17. Chou R, Selph S, Buckley DI, et al. Comparative effectiveness of fluorescent versus white light cystoscopy for initial diagnosis or surveillance of bladder cancer on clinical outcomes: systematic review and meta-analysis. J Urol 2017;197:548-58.

18. Rolevich AI, Zhegalik AG, Mokhort AA, et al. Results of a prospective randomized study assessing the efficacy of fluorescent cystoscopy-assisted transurethral resection and single instillation of doxorubicin in patients with non-muscle-invasive bladder cancer. World J Urol 2017;35:745-52.

19. Bolenz C, Rother J, Meessen S, et al. The development of real-time multispectral imaging for the diagnostics of bladder cancer. Urologe A 2019;58:1435-42.

20. Kriegmair MC, Rother J, Grychtol B, et al. Multiparametric Cystoscopy for Detection of Bladder Cancer Using Real-time Multispectral Imaging. Eur Urol 2020;77:251-9.

21. Dimitriadis N, Grychtol B, Theuring M, et al. Spectral and temporal multiplexing for multispectral fluorescence and reflectance imaging using two color sensors. Opt Express 2017;25:12812-29.

Cite this article as: Grüne B, Rother J, Waldbillig F, Chellappan G, Meessen S, Grychtol B, Deliolanis NC, Bolenz C, Kriegmair MC. Ex vivo validation of a real-time multispectral endoscopic system for the detection and biopsy of bladder tumors. Transl Androl Urol 2021;10(6):2373-2383. doi: $10.21037 /$ tau-20-1372
22. Ahmadi H, Daneshmand S. Multiparametric cystoscopy: is the future here yet? Transl Androl Urol 2021;10:1-6.

23. Brooke J. SUS: A quick and dirty usability scale. Usability Evaluation in Industry 1996;189:47.

24. Bangor A, Kortum P, Miller J. Determining what individual SUS scores mean: adding an adjective rating scale. J Usability Stud 2009;4:114-23.

25. Sonn GA, Mach KE, Jensen K, et al. Fibered confocal microscopy of bladder tumors: an ex vivo study. J Endourol 2009;23:197-201.

26. Pan YT, Xie TQ, Du CW, et al. Enhancing early bladder cancer detection with fluorescence-guided endoscopic optical coherence tomography. Opt Lett 2003;28:2485-7.

27. Cordero E, Rüger J, Marti D, et al. Bladder tissue characterization using probe-based Raman spectroscopy: evaluation of tissue heterogeneity and influence on the model prediction. J Biophotonics 2020;13:e201960025.

28. Waldbillig F, von Rohr L, Nientiedt M, et al. Preclinical and clinical evaluation of a novel, variable-view, rigid endoscope for female cystoscopy. Urology 2020;142:231-6.

29. Waldbillig F, von Rohr L, Nientiedt M, et al. Endourological training using 3D-printed bladder phantoms: development and prospective evaluation. J Endourol 2021. [Epub ahead of print]. doi: 10.1089/ end.2020.0900.

30. Poletajew S, Krajewski W, Gajewska D, et al. Prediction of the risk of surgical complications in patients undergoing monopolar transurethral resection of bladder tumour - a prospective multicentre observational study. Arch Med Sci 2019;16:863-70. 